\title{
Kommentar zum jüngsten Suizidhilfe-Entscheid des Europäischen Gerichtshofs für Menschenrechte
}

\author{
Das schweizerische Recht sagt nicht genügend klar, in welchen Fällen Suizidhilfe er- \\ laubt ist. Aus diesem Grund hat der Europäische Gerichtshof für Menschenrechte \\ eine Verletzung der Europäischen Menschenrechtskonvention durch die Schweiz \\ festgestellt. Dieser Beitrag erörtert die rechtlichen Implikationen dieses Urteils. Der \\ folgende Beitrag von Alex Mauron betrachtet die ethischen Implikationen.
}

\section{Daniel Hürlimann}

Dr. iur., Rechtsanwalt, CAS Judikative, Forschungsmitarbeiter im Nationalen Forschungsprogramm «Lebensende» (www.nfp67.ch) des Schweizerischen Nationalfonds an den Universitäten Luzern und Zürich*

Der Autor dankt Prof. Dr. iur. Regina Kiener, Prof. Dr. iur. Bernhard Rütsche, lic. phil. MLaw Lea Schläpfer und Dr. med. Anna K. Stuck für die kritische Durchsicht des Manuskripts.

\footnotetext{
* Der Bundesrat hat am 1. Mai 2013 in Beantwortung einer parlamentarischen Anfrage festgehalten, vom NFP 67 werde erwartet, dass «die heutige Praxis der Sterbehilfe untersucht und gegebenenfalls Empfehlungen und Änderungsvorschläge ausgearbeitet werden».

** Die Referenzen finden sich unter www.saez.ch $\rightarrow$ Aktuelle Nummer oder $\rightarrow$ Archiv $\rightarrow 2013 \rightarrow 31 / 32$.
}

Korrespondenz:

Dr. iur. Daniel Hürlimann

Universität Luzern

Rechtswissenschaftliche

Fakultät

Frohburgstrasse 3

Postfach 4466

CH-6002 Luzern

daniel.huerlimann[at]unilu.ch

\section{Vorgeschichte}

«Eine deutliche Mehrheit der Kantone, Parteien und interessierten Organisationen hat sich in der Vernehmlassung für eine ausdrückliche Regelung der organisierten Suizidhilfe auf Bundesebene ausgesprochen.» Dieser Satz stammt aus einer Medienmitteilung des Eidgenössischen Justiz- und Polizeidepartements (EJPD) aus dem Jahr 2010, nachdem das Vernehmlassungsverfahren zu einer Neuregelung der Suizidhilfe im Strafgesetzbuch abgeschlossen war $[1]^{* *}$. Gestützt darauf beauftragte der Bundesrat das Departement, bis Ende 2010 eine Botschaft mit Gesetzesentwurf auszuarbeiten. Im Juni 2011 folgte sodann die Kehrtwende, indem der Bundesrat entschied, auf eine Regelung der organisierten Suizidhilfe im Strafrecht $\mathrm{zu}$ verzichten [2]. Was war geschehen?

Abgesehen von Änderungen in der personellen Zusammensetzung des Bundesrates und auch an der Spitze des EJPD ergab schon die Vernehmlassung, dass eine Regelung zwar von einer deutlichen Mehrheit befürwortet wurde, dass man sich aber über den Inhalt einer solchen alles andere als einig war. Zur Auswahl standen ein absolutes Verbot der organisierten Suizidhilfe [3], was mit Blick auf die Gefühlslage im Volk von vornherein kaum umsetzbar erschien, sowie eine detaillierte Regelung der Voraussetzungen für die Straflosigkeit [4]. Wie bereits in früheren Anläufen hatte sich der Bundesrat auf das Strafrecht beschränkt und kam zum Ergebnis, dass die vorgeschlagene Bestimmung lediglich die Verpflichtungen konkretisiert hätte, die sich ohnehin aus dem geltenden Recht ergeben (Art. 115 StGB). Daran ist nichts auszusetzen.

Eine andere Frage ist jedoch, ob das Strafrecht das richtige Gefäss für eine Regulierung der organisierten Suizidhilfe ist. Die Regeln, die das Bundesgericht bisher angewendet hat, stammen von der Schweizerischen Akademie der Medizinischen Wissenschaften (SAMW). Es handelt sich dabei nicht um staatliches Recht, sondern um Standesrecht. Obwohl diese Richtlinien in der Schweiz - so weit ersichtlich - relativ

\section{Commentaire sur le dernier arrêt} de la Cour européenne des droits de l'homme relatif à l'assistance

\section{au suicide}

La volonté de réglementer l'assistance au suicide n'est pas nouvelle, mais sa concrétisation a jusqu'ici échoué notamment parce que l'on s'est contenté d'aborder cette question sous l'angle du droit pénal. De ce fait, les bases légales actuellement en vigueur comportent des lacunes et laissent certaines questions en suspens, en particulier celle de savoir si l'assistance au suicide est également admise pour les personnes qui ne se trouvent pas en situation de fin de vie. Forte de cette constatation, la Cour européenne des droits de l'homme (CEDH) a demandé à la Suisse d'élaborer des directives claires et exhaustives concernant l'accès de ces personnes à une substance mortelle.

breit akzeptiert sind, wirft die Regelung eines so zentralen Themas durch (demokratisch nicht legitimiertes) Standesrecht grundsätzliche Fragen auf. Da sich die SAMW-Richtlinien auf die Suizidhilfe am Lebensende beschränken, bleibt zudem unklar, wie mit dem Suizidwunsch von Personen umzugehen ist, die sich nicht am Lebensende befinden.

\section{Urteil des EGMR}

Genau dieses Problem hat sich nun im Fall manifestiert, der vor wenigen Wochen durch den Europäischen Gerichtshof für Menschenrechte (EGMR) entschieden wurde: Eine 82-jährige urteilsfähige Frau möchte seit mindestens acht Jahren sterben, befindet sich aber unbestrittenermassen noch nicht am Le- 


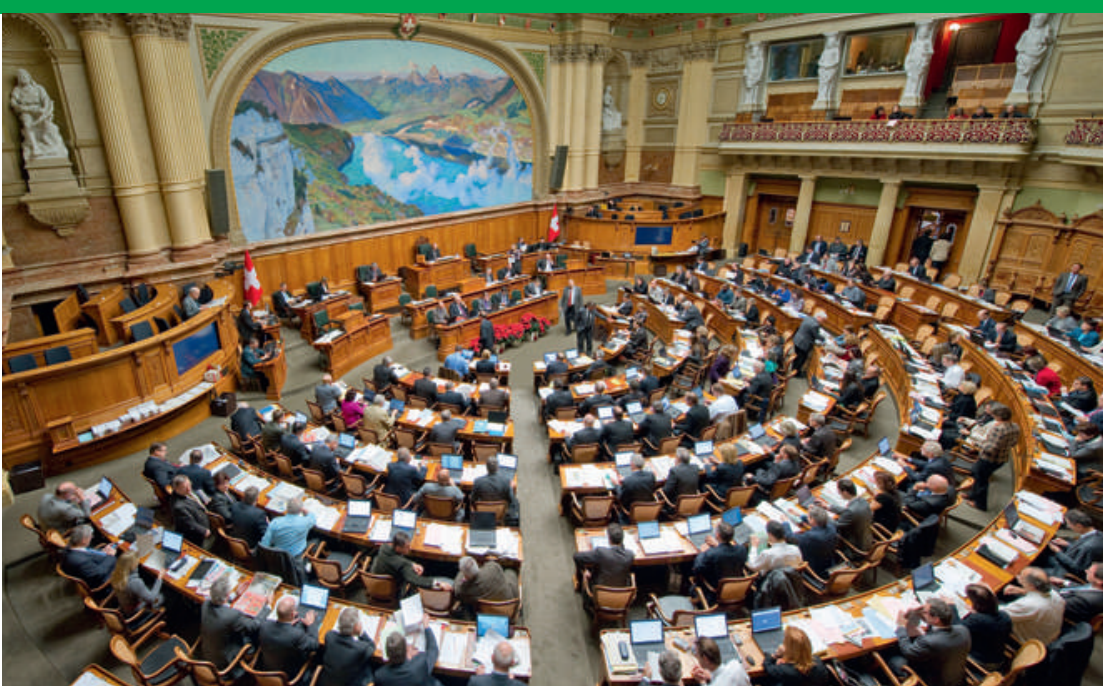

Eine Anpassung der gesetzlichen Regelungen zur Suizidhilfe durch den Bundesrat ohne Mitwirkung des Parlaments dürfte keine Lösung sein.

bensende im Sinne der SAMW-Richtlinien. Mehrere Ärzte lehnten die Verschreibung des todbringenden Mittels (Natrium-Pentobarbital) nicht aus Gewissensgründen $\mathrm{ab}$, sondern weil sie standesrechtliche Konsequenzen befürchteten. Die Frau gelangte in der Folge an die Gesundheitsdirektion des Kantons Zü-

\section{Beanstandet wurden das Fehlen jeglicher Anhaltspunkte} für den Umgang mit solchen Fällen und die daraus resultierende Unsicherheit für die Betroffenen.

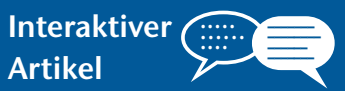

Wollen Sie diesen Artike kommentieren? Nutzen Sie dafür die Kommentarfunktion in der OnlineVersion oder sehen Sie nach, was Ihre Kolleginnen und Kollegen bereits geschrieben haben: www.saez.ch/ aktuelle-ausgabe/ interaktive-beitraege/ rich, welche die Verschreibung ebenfalls ablehnte und deren Entscheid vom Zürcher Verwaltungsgericht [5] und vom Bundesgericht [6] bestätigt wurde. Zuletzt wandte sie sich an den EGMR, machte eine Verletzung ihres (gestützt auf ein früher ergangenes Urteil des EGMR [7] bestehenden) Rechts auf Wahl von Art und Zeitpunkt der Lebensbeendigung geltend und verlangte die Verschreibung des NatriumPentobarbitals.

In seinem Urteil vom 14. Mai 2013 erkennt der EGMR eine Verletzung von Artikel 8 der Europäischen Menschenrechtskonvention [8] (Recht auf Achtung des Privat- und Familienlebens) durch die Schweiz, indem diese nicht genügend klare Leitlinien im Hinblick auf den Umfang des Rechts auf Lebensbeendigung bereitstellt [9]. Dies habe dazu geführt, dass sich die Beschwerdeführerin in einem Zustand der Angst und Ungewissheit wiedergefunden habe [10].

Der Gerichtshof äussert sich explizit nicht dazu, wie eine allfällige Regulierung aussehen soll [11], und lässt damit grundsätzlich auch die Möglichkeit offen, für solche Fälle eine Verschreibung zu verbieten [12]. Die Schweiz verletzt gemäss Urteilstext die Menschenrechtskonvention einzig dadurch, dass sie bis heute keine genügend klare Regulierung für solche Fälle erlassen hat. Dies ist - entgegen der Bericht- erstattung in der NZZ vom 15. Mai 2013 [13] - nicht als Kampfansage an das Richterrecht zu verstehen: Beanstandet wurde nämlich nicht etwa eine fallorientierte Entscheidungsfindung, sondern das Fehlen jeglicher Anhaltspunkte für den Umgang mit solchen Fällen und die daraus folgende Unsicherheit für die betroffenen Menschen, aber auch für weitere Akteure wie namentlich die mitwirkenden Ärztinnen und Ärzte.

Der Gerichtshof hat es darüber hinaus auch abgelehnt, eine Verschreibung von Natrium-Pentobarbital im konkreten Fall anzuordnen [14]. Stattdessen hat er darauf hingewiesen, dass es das Ziel der Europäischen Menschenrechtskonvention sei, die darin festgeschriebenen Rechte und Freiheiten durch die Mitgliedstaaten und deren Rechtssysteme zu gewährleisten.

\section{Rechtsfolgen des Urteils}

Zunächst ist darauf hinzuweisen, dass die Schweiz eine Verweisung an die Grosse Kammer des EGMR beantragt hat [15]. Der Antrag wird angenommen, wenn die Rechtssache nach Auffassung eines fünfköpfigen Ausschusses eine schwerwiegende Frage von allgemeiner Bedeutung aufwirft. In diesem Fall bliebe abzuwarten, wie die Grosse Kammer mit 17 Richtern entscheidet. Ansonsten ist die Schweiz verpflichtet, das Urteil des Gerichtshofes vom 14. Mai $2013 \mathrm{zu}$ befolgen.

An dieser Stelle soll nicht darüber spekuliert werden, wie die Grosse Kammer entscheiden könnte. Es sei lediglich darauf hingewiesen, dass das Urteil mit $4 \mathrm{zu} 3$ Stimmen gefällt worden ist und dass die Schweizer Richterin mit der Mehrheit gestimmt hat. Unbestritten dürfte sein, dass es zur Frage der Regulierung der (organisierten) Suizidhilfe eine gesamtgesellschaftliche Diskussion braucht. Diese ist jetzt mit dem - nur schon deshalb zu begrüssenden - Urteil des EGMR wieder lanciert. Indem der EGMR auf die Schwierigkeiten der politischen Konsensfindung in ethischen Fragen verweist [16], deutet er an, dass die Entwicklung von Leitlinien durch das Bundesgericht nicht genügen dürfte und dass es eine gesetzliche Regelung braucht.

Auch wenn der Europäische Gerichtshof für Menschenrechte offenlässt, ob ein Gesetz im formellen Sinn (und nicht nur eine Verordnung) erlassen werden muss, ist festzuhalten, dass eine Anpassung durch den Bundesrat ohne Mitwirkung des Parlaments [17] keine Lösung sein kann. Eine allfällige Liberalisierung der Regeln für die Verschreibung von Natrium-Pentobarbital bedürfte einer Änderung des Heilmittelgesetzes [18], das verlangt, dass beim Umgang mit Heilmitteln die Gesundheit des Menschen nicht gefährdet wird. Die Verschreibung einer tödlichen Dosis Natrium-Pentobarbital widerspricht somit den allgemeinen Bestimmungen des aktuell gültigen Gesetzes [19]. 


\section{Referenzen}

1 Medienmitteilung des Eidgenössischen Justiz- und Polizeidepartements vom 17. September 2010, abrufbar unter www.news.admin.ch/message/?msg-id=35187.

2 Medienmitteilung des Bundesrates vom 29. Juni 2011, abrufbar unter www.news.admin.ch/message/ ?msg-id=39905

3 Vorentwurf/Variante 2, Art. 115 StGB «Wer aus selbstsüchtigen Beweggründen oder im Rahmen einer Suizidhilfeorganisation jemanden zum Suizid verleitet oder ihm dazu Hilfe leistet, wird, wenn der Suizid ausgeführt oder versucht wird, mit Freiheitsstrafe bis zu fünf Jahren oder Geldstrafe bestraft.»

4 Vgl. die einzelnen Voraussetzungen im Vorentwurf/ Variante 1 (beide Varianten abrufbar unter www.lebensende.ch/gesetzgebung/stgb-ve2009).

5 Endentscheid des Verwaltungsgerichts des Kantons Zürich vom 22. Oktober 2009, abrufbar unter www.lebensende.ch/urteile/vgrzh/nap-abgabe.

6 Urteil des Bundesgerichts 2C_9/2010 vom 12. April 2010, abrufbar unter www.lebensende.ch/urteile/bger/ nap-abgabe.

7 Urteil des EGMR vom 20. Januar 2001 (Haas gegen Schweiz, abrufbar unter www.lebensende.ch/urteile/ egmr/haas-v-ch), Ziff. 51: «In the light of this case-law, the Court considers that an individual's right to decide by what means and at what point his or her life will end, provided he or she is capable of freely reaching a decision on this question and acting in consequence, is one of the aspects of the right to respect for private life within the meaning of Article 8 of the Convention.»

8 Konvention vom 4 . November 1950 zum Schutze der Menschenrechte und Grundfreiheiten (EMRK)

9 Urteil des EGMR vom 14. Mai 2013 (Gross gegen Schweiz, abrufbar unter www.lebensende.ch/urteile/ egmr/gross-v-ch), Ziff. 67: «The foregoing considerations are sufficient to enable the Court to conclude that Swiss law, while providing the possibility of obtaining a lethal dose of sodium pentobarbital on medical prescription, does not provide sufficient guidelines ensuring clarity as to the extent of this right. There has accordingly been a violation of Article 8 of the Convention in this respect.»

10 Urteil des EGMR vom 14. Mai 2013, Ziff. 66: «The Court concludes that the applicant must have found herself in a state of anguish and uncertainty regarding the extent of her right to end her life which would not have occurred if there had been clear, State-approved guidelines $[. .$.$] .»$

11 Urteil des EGMR vom 14. Mai 2013, Ziff. 69: «Accordingly, the Court decides to limit itself to the conclusion that the absence of clear and comprehensive legal guidelines violated the applicant's right to respect for her private life under Article 8 of the Convention, without in any way taking up a stance on the substantive content of such guidelines.»
12 Urteil des EGMR vom 29. April 2002 (Pretty gegen das Vereinigte Königreich, abrufbar unter www.lebensende.ch/urteile/egmr/pretty-v-uk), Ziff. 67: «The Court is not prepared to exclude that this constitutes an interference with her right to respect for private life as guaranteed under Article 8 \& 1 of the Convention.»

13 NZZ vom 15. Mai 2013, S. 10 («Strassburg will Gesetzesregelung», abrufbar unter www.lebensende.ch/zeitung/ nzz/gross-v-ch)

14 Urteil des EGMR vom 14. Mai 2013, Ziff. 68 f.

15 Art. 43 EMRK (Verweisung an die Grosse Kammer).

16 Urteil des EGMR vom 14. Mai 2013, Ziff. 66: «The Court acknowledges that there may be difficulties in finding the necessary political consensus on such controversial questions with a profound ethical and moral impact. However, these difficulties are inherent in any democratic process and cannot absolve the authorities from fulfilling their task therein.»

17 Die Änderung einer Verordnung (z. B. Betäubungsmittelkontrollverordnung) ist ohne Mitwirkung des Parlaments möglich.

18 Bundesgesetz vom 15. Dezember 2000 über Arzneimittel und Medizinprodukte (Heilmittelgesetz, HMG)

19 In diesem Zusammenhang sei auf das Urteil des Tribunal régional du Littoral et du Val-de-Travers vom 8. Juli 2013 verwiesen (abrufbar unter www.lebensende.ch/urteile/boudry-ne/nap-abgabe), in dem ein Arzt gestützt auf Art. 86 i.V.m. Art. 26 Abs. 2 HMG für die Verschreibung von Natrium-Pentobarbital zu einer Busse von 500 Franken verurteilt wurde; vgl. Der Bund vom 9. Juli 2013, S. 22 («Neuenburger Arzt wegen Sterbehilfe verurteilt»). Der Arzt hatte beim Patienten zu Hause festgestellt, dass dieser wahrscheinlich an einer anorektalen Tumorerkrankung leide. Acht Tage später hat er das Rezept für eine tödliche Dosis Natrium-Pentobarbital ausgestellt, und am darauffolgenden Tag ist der Patient nach dessen Einnahme gestorben. Der Schuldspruch erfolgte wegen Verstosses gegen Art. 26 Abs. 2 HMG, wonach ein Arzneimittel nur verschrieben werden darf, «wenn der Gesundheitszustand der Konsumentin oder des Konsumenten beziehungsweise der Patientin oder des Patienten bekannt ist.» Das Gericht erachtete diese Voraussetzung als nicht erfüllt, weil der Arzt nur einen Einblick («aperçu») und keine Kenntnis («connaissance») des Zustands seines Patienten hatte. Dessen Beschwerden seien nicht zweifelsfrei die Folge einer anorektalen Tumorerkrankung gewesen, sondern könnten nach Einschätzung von zwei anderen Ärzten ihre Ursache auch in einer Entzündung gehabt haben. Schliesslich äussert sich das Gericht zur Frage, ob eine Verurteilung gestützt auf die jüngste Rechtsprechung des EGMR ausgeschlossen sei. Es verneint dies mit der folgenden Argumentation: Entweder sei der vorliegende Fall mit dem Sachverhalt im Verfahren «Gross gegen Schweiz» vergleichbar, dann bräuchte es die vom EGMR verlangten Leitlinien und diese könnten nicht durch das Gericht erahnt oder erlassen werden. Oder aber der Fall sei gar nicht vergleichbar, weil sich im Verfahren vor dem EGMR die betroffene Person eindeutig nicht am Lebensende befunden habe. 